\title{
Rollover Mitigation Controller Development for Three-Wheeled Vehicle Using Active Front Steering
}

\author{
Raja Amer Azim, Fahad Mumtaz Malik, and Waheed ul Haq Syed \\ NUST College of Electrical \& Mechanical Engineering, National University of Sciences \& Technology, Peshawar Road, \\ Rawalpindi 46000, Pakistan
}

Correspondence should be addressed to Raja Amer Azim; raja_amer@ceme.nust.edu.pk

Received 29 August 2014; Revised 12 January 2015; Accepted 23 January 2015

Academic Editor: Sabri Arik

Copyright (C) 2015 Raja Amer Azim et al. This is an open access article distributed under the Creative Commons Attribution License, which permits unrestricted use, distribution, and reproduction in any medium, provided the original work is properly cited.

\begin{abstract}
Three-wheeled vehicles are agile, less complex, but relatively more prone to rollover. The current study focuses on the rollover mitigation control design using active front steering for such vehicles. A lateral load transfer ratio (LLTR) adapted for a threewheeled platform is presented. Sliding mode control design strategy has been devised which results in pseudo-direct control for roll dynamics of the vehicle. The lag in vehicle roll angle response has been managed using adaptive sliding surface. This concept can be extended for other vehicle configurations. The proposed control scheme is investigated for efficacy using a full vehicle simulation model of CarSim software and National Highway Traffic Safety Administration's proposed Fishhook maneuver. The controller is able to limit the rollover propensity even with vehicle parameter uncertainties.
\end{abstract}

\section{Introduction}

Typical aircrafts while taxing on ground, many wheeled robots, and some off- and on-road vehicles use a threewheeled configuration. Due to agility and weight benefits, it is also used as a transport medium for short distance public transport. In wheeled vehicles, rollover accidents are a very small fraction of the total accidents but account for more than one third of the total fatalities and severe injuries [1]. Static stability factor is one of the benchmarks used by National Highway Traffic Safety Administration (NHTSA) for comparison of relative rollover likelihood of vehicles. It is the ratio between the track width and height of center of gravity (cg) [2]. For a three-wheeled vehicle this ratio is reduced as compared to a four-wheeled vehicle of similar size, by a factor equal to the dimensionless distance of $\mathrm{cg}$ from rear axle [3]. Hence, with the same track width and cg height, a three-wheeled configuration is more prone to rollover than a similar sized four-wheeled platform. Roll dynamics of a three-wheeled vehicle is sensitive to the vehicle track width, height, and longitudinal position of cg as observed in [3-5]. Mukherjee et al. [6] evaluated the rollover propensity of a three-wheeled vehicle using J-Turn and Road Edge Recovery
(RER) maneuvers. The wheel liftoff condition was used as the rollover threshold. They found that the maneuver entry speed at nominal loading conditions, which corresponds to wheel liftoff, was $7.98 \mathrm{~m} / \mathrm{s}$ for J-Turn and $9.0 \mathrm{~m} / \mathrm{s}$ for RER. On the other hand the maneuver entry speed for J-Turn and RER for a four-wheeled (SUV) vehicle in reduced rollover configuration was found to be $17.29 \mathrm{~m} / \mathrm{s}$ and $16.09 \mathrm{~m} / \mathrm{s}$, respectively, as reported by Forkenbrock et al. [7]. Hence, the rollover susceptibility of a three-wheeled vehicle is almost two times more than that of a least stable four-wheeled SUV. Based upon lateral acceleration limits for rollover, four-wheeled vehicles appear more prone to directional instabilities than rollover as compared to three-wheeled vehicles.

Rollover of three-wheeled vehicles has resulted in serious injuries and even fatalities of passengers. On the average, of the total traffic related fatalities reported in India in 2009 2011 [8-10], three-wheeled vehicles were involved in $5 \%$ of the total accidents. The share in serious injuries is $8 \%$. Even with these limitations, production of three-wheeled vehicle has doubled in India. In Mumbai three-wheeled vehicles are $11 \%$ of the total vehicles, whereas $20 \%$ of daily commuters use three-wheeled vehicles [11]. This highlights an increased risk as compared to other forms of transport. 
Another important factor revealed by a report on accident data [8] was that $77.5 \%$ of reported accidents were due to driver's mistake. Conventional three-wheeled vehicles have a $1: 1$ steering and road-wheel angle ratio. This results in very high rate steering input in emergency, or other impulsive reactions by the driver. The steering response of threewheeled vehicles is also significantly fast as compared to fourwheeled vehicles $[3,12]$, thus highlighting its vulnerability.

Rollover events are classified as tripped and untripped [13]. Abrupt bumps and soft soil patches induce tripped rollovers. These rollovers may occur even while traveling on a straight path. Maneuver induced rollovers are termed as untripped rollovers which are mainly the result of improper driver input, that is, steering and speed while cornering. NHTSA in report [14] highlights that ESC (Electronic Stability Control) systems reduce fatalities by $34 \%$ in multiple vehicle crashes for passenger cars and SUVs. Single vehicle crashes resulted in up to $74 \%$ less fatalities in SUVs using ESC systems. This signifies the importance and efficacy of such systems and therefore a considerable research has focused on this area [15-18].

Untripped rollover prevention systems can be classified into three main categories. The first type utilizes active suspension systems which directly controls the roll motion resulting in increased rollover threshold [19-21]. The second category is based upon the roll and yaw motion coupling. Braking to reduce speed, differential braking, and steering are employed, which indirectly reduces lateral acceleration to manage vehicle roll motion [22-24]. The third category is termed as integrated chassis control which is usually the combination of direct and indirect control interventions [2527]. Active suspension systems are typically expensive and braking control relies on existing antilock braking system (ABS). All these systems have been developed for four or more wheeled vehicles with wheels on both sides of the longitudinal mid-plane.

In a typical one-front and two-rear wheel (delta) configuration, a three-wheeled vehicle has no roll stiffness at the front axle. The rear two-wheeled axle unloads in braking, which reduces the lateral load transfer margin for rollover. Because of this difference in vehicle setup, the above mentioned control systems have not yet been incorporated for commercial three-wheeled vehicles. For three-wheeled platforms, active leaning mechanisms for rollover mitigation have been proposed and developed as one of the solutions to address this problem [28-30]. Two main schemes for tilt control exist. One is the Direct Tilt Control (DTC) as proposed by Piyabongkarn et al. [28]. In this scheme dedicated actuators were used for tilting the vehicle based on lateral acceleration and input steering angle. The whole vehicle is controlled to lean towards the turn center. In order to effectively mitigate rollover threat, it requires significant actuator effort to initiate leaning. To overcome this problem, a split chassis design using steering tilt control (STC) was proposed by Barker et al. [29], which incorporates leaning of front single wheel for steering and passenger compartment while the rear engine pod with actuators does not lean. A combination of both STC and DTC modes was adopted by Kidane et al. [30] with an additional tilt brake system.
The proposed techniques for three-wheeled vehicles discussed above though effective have significant limitations from implementation perspective for a single unit chassis commercial three-wheeled vehicle. The current study focuses on a rollover prevention system for existing single chassis three-wheeled platforms. Considering the limitations of a brake based system a steering based rollover prevention system is proposed. A superposition (additive) steering can be integrating into existing system making it a viable addon. A rollover mitigation/prevention controller is designed using sliding mode control based on a lower order full vehicle roll dynamics model. The model has the advantage that the steering input directly affects the roll motion. Such low fidelity models represent pre-liftoff roll dynamics with reasonable accuracy for four-wheeled vehicles as discussed in $[31,32]$. To conserve power a control trigger rule was used. CarSim and Simulink environments are set for cosimulation to evaluate the dynamics of the vehicle and performance of the controller under NHTSA defined Fishhook maneuvers. Effect of vehicle mass properties and cg height variation on the controller performance was investigated.

The paper is organized as the following: vehicle model is presented in Section 2, lateral load transfer ratio adapted for a three-wheeled vehicle is presented in Section 3, the proposed controller is detailed in Section 4 followed by simulations in Section 5, and conclusions are presented in Section 6.

\section{Vehicle Model for Controller Design}

In this study, a full vehicle model adapted from [4] is used. Small angle approximations were used neglecting unsprung mass and roll axis effects. In a typical rollover controller design, roll-yaw motion coupling is exploited to control roll dynamics. However the employed model enables direct control of roll motion using steering input. The modeling scheme used also facilitates finding equivalent control.

A rigid chassis model with six degrees of freedom is used. Unsprung mass is lumped with the sprung mass.

Using a vehicle fixed coordinate system at the center of gravity as shown in Figure 1, equations of motions are derived as given below:

$$
\begin{gathered}
\ddot{x}=\dot{y} \dot{\psi}+\frac{P(t)}{m \dot{x}}-\frac{\mu_{r}}{m}\left(F z_{r l}+F z_{r r}+F z_{f}\right) \\
-\frac{\left(B x_{r l}+B x_{r r}+B x_{f}\right)}{m}, \\
\ddot{y}=\dot{x} \dot{\psi}+\frac{\left(F y_{f}+F y_{r l}+F y_{r r}\right)}{m}, \\
\ddot{z}=-g+\frac{\left(F z_{f}+F z_{r l}+F z_{r r}\right)}{m}-\frac{\dot{\phi} \dot{y}}{m}, \\
\ddot{\phi}=\frac{1}{I_{x x}}\left[b\left(F z_{r l}-F z_{r r}\right)-\left(h_{0}+z\right)\left(F y_{f}+F y_{r l}+F y_{r r}\right)\right. \\
\left.-\dot{\theta} \dot{\psi}\left(I_{z z}-I_{y y}\right)\right],
\end{gathered}
$$




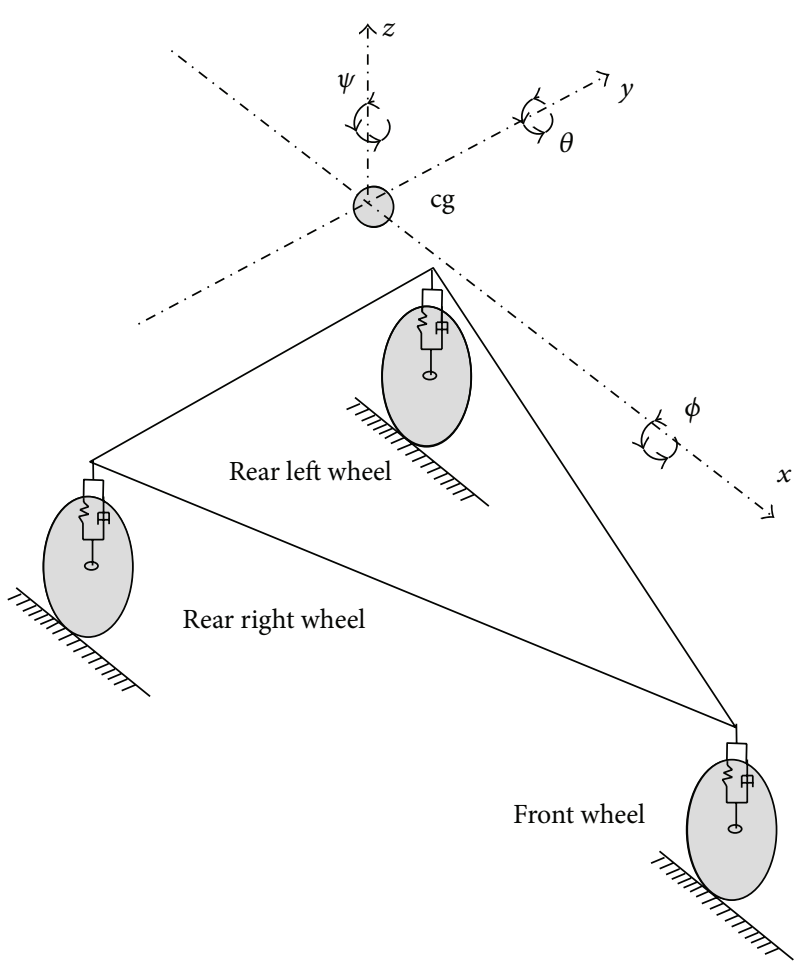

Figure 1: Vehicle layout used for model development. The equations of motion are derived with respect to the coordinate system placed at the center of gravity.

$$
\begin{aligned}
\ddot{\psi}=\frac{1}{I_{z z}}[ & b \mu_{r}\left(F z_{r r}-F z_{r l}\right)+b\left(B x_{r r}-B x_{r l}\right) \\
& \left.+F y_{f} l_{f}-l_{r}\left(F y_{r l}+F y_{r r}\right)-\dot{\phi} \dot{\theta}\left(I_{y y}-I_{z z}\right)\right], \\
\ddot{\theta}=\frac{1}{I_{y y}} & {\left[l_{f} F z_{f}-l_{r}\left(F z_{r l}+F z_{r r}\right)\right.} \\
& \left.+\left(h_{0}+z\right)\left(\frac{P(t)}{\dot{x}}-\mu_{r} \sum F z-\sum B x\right)\right] .
\end{aligned}
$$

Linear tire model is used for calculating lateral forces as follows:

$$
\begin{aligned}
& F y_{f}=C_{\alpha f} \alpha_{f}, \\
& F y_{r l}=C_{\alpha r} \alpha_{r l}, \\
& F y_{r r}=C_{\alpha r} \alpha_{r r},
\end{aligned}
$$

where $C_{\alpha r}$ and $C_{\alpha f}$ are rear and front cornering stiffness; and the slip angles $\alpha_{f}, \alpha_{r l}, \alpha_{r r}$ are expressed in terms of vehicle states as follows:

$$
\begin{aligned}
& \alpha_{f}=\delta_{f}-\frac{\left(\dot{y}+l_{l} \dot{\psi}\right)}{\dot{x}}, \\
& \alpha_{r l}=-\frac{\dot{y}-l_{r} \dot{\psi}}{\dot{x}-b \dot{\psi}}, \\
& \alpha_{r r}=-\frac{\dot{y}-l_{r} \dot{\psi}}{\dot{x}+b \dot{\psi}},
\end{aligned}
$$

where $\delta_{f}$ is the front steering angle. The vertical forces on each wheel are calculated as follows:

$$
\begin{aligned}
& F z_{f}=F z_{f i}+K_{f}\left(-z+l_{f} \theta\right)+c_{f}\left(-\dot{z}+l_{f} \dot{\theta}\right), \\
& F z_{r l}=F z_{r l i}+K_{r}\left(-z-l_{r} \theta-b \phi\right)+c_{r}\left(-\dot{z}-l_{r} \dot{\theta}-b \dot{\phi}\right), \\
& F z_{r r}=F z_{r r i}+K_{r}\left(-z-l_{r} \theta+b \phi\right)+c_{r}\left(-\dot{z}-l_{r} \dot{\theta}+b \dot{\phi}\right),
\end{aligned}
$$

where subscript $i$ denotes initial static forces; $K$ is the effective suspension stiffness and $c$ is the effective suspension damping as tire characteristics are lumped with the suspension compliance characteristics. It may be noted here that the $-l_{r} \theta$ and $-l_{r} \dot{\theta}$ terms unload the rear wheels in breaking and hence reduce the total lateral load transfer required to unload the rear wheels which is further defined in Section 3 of this paper.

The roll dynamics equation in terms of state variables can be written as follows:

$$
\begin{aligned}
\ddot{\phi}=\frac{1}{I_{x x}}\{ & (h+z)\left[-\frac{2 \dot{x}\left(\dot{y}-l_{r} \dot{\psi}\right) C_{\alpha r}}{\left(\dot{x}^{2}-b^{2} \dot{\psi}^{2}\right)}\right] \\
& -2 b^{2}\left(K_{r} \phi+c_{r} \dot{\phi}\right) \\
& -(h+z)\left[\frac{\left(\dot{y}+l_{f} \dot{\psi}\right) C_{\alpha f}}{\dot{x}}\right] \\
& \left.-\dot{\theta} \dot{\phi}\left(I_{z z}-I_{y y}\right)\right\}+\frac{(h+z)}{I_{x x}} \delta_{f} .
\end{aligned}
$$

Nominal values for parameters $l_{r}, l_{f}, b, m, l$ are directly used from previous studies on three-wheeled vehicle [4, $5,33,34]$, while the parameters $C_{\alpha f}, C_{\alpha r}, h$ are adapted by minimizing a cost function $J$ as given in (8), which is the summation of normalized errors of roll angle and roll rate between values obtained from (7) and CarSim. This ensures that the model used for controller design represents vehicle behavior with reasonable accuracy:

$$
J=\sum\left(\frac{\left|\phi_{\text {CarSim }}-\phi_{\text {Model }}\right|}{\max \left|\phi_{\text {CarSim }}\right|}+\frac{\left|\dot{\phi}_{\text {CarSim }}-\dot{\phi}_{\text {Model }}\right|}{\max \left|\dot{\phi}_{\text {CarSim }}\right|}\right)
$$

The response in terms of roll angle and roll rate to a step input of 3 degrees at $40 \mathrm{~km} / \mathrm{hr}$ speed is shown in Figure 2.

The response clearly indicates a delay of almost 0.55 seconds for the roll angle to evolve. Although close to a nominal step input of this type is uncommon in typical road vehicles due to the rate at which an average person can turn the wheel coupled with the reduction in steering angle input from hand-wheel to the road wheel. The steering input rate used in NHTSA J-turn test is $720 \mathrm{deg} / \mathrm{sec}$, which effectively translates into 36 degrees per second for a typical 20:1 reduction. In the absence of any reduction in the steering system of a common three-wheeled vehicle using motorcycle steering assemblies, a steering input close to step input is in fact a typical input for an evasive maneuver. 


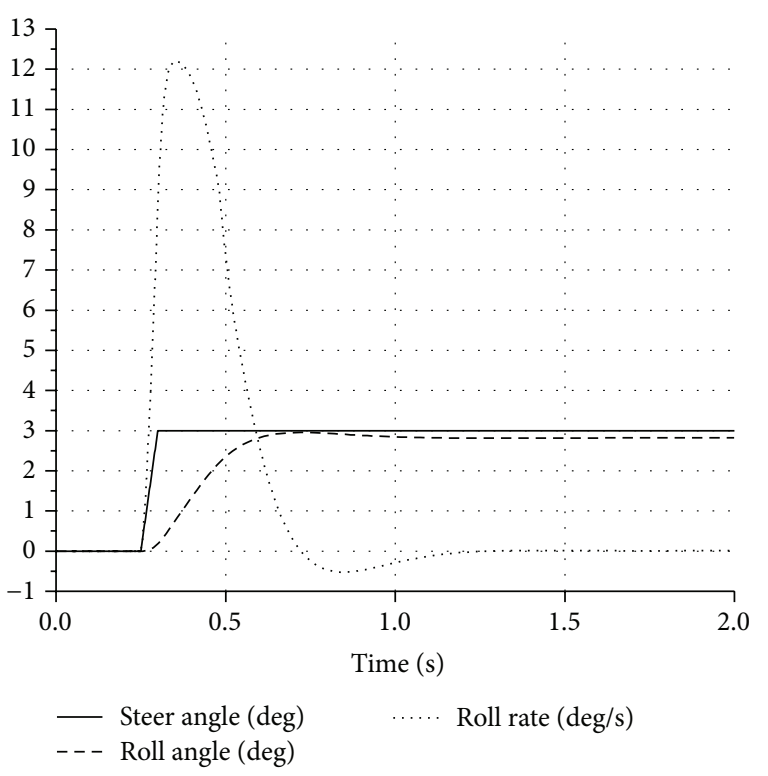

FIGURE 2: Roll angle and roll rate of vehicle resulting from a step steering input of 3 degrees.

\section{Rollover Detection}

During cornering, the inner wheel(s) in a turn are unloaded and the load is transferred to the outer wheel(s). NHSTA defines liftoff when all wheels of one side of a vehicle leave contact with the ground. This loss of contact means lesser influence of driver input and loss of roll damping provided by the suspension. It is for this reason that wheel liftoff condition is taken as the threshold or limiting value for all control scheme developments. Several factors such as track width, height of center of gravity, and suspension characteristics affect the rate at which this load transfer occurs; hence specific rollover propensity for a particular vehicle setup exists as elaborated in [35-37]. The lateral load transfer ratio (LLTR) is given by (9) as in [25]:

$$
\text { LLTR }=\frac{F z_{r}-F z_{l}}{\left|F z_{r}+F z_{l}\right|},
$$

where $F z_{r}$ and $F z_{l}$ are the total vertical forces on the right and left side wheels. The value varies from -1 to 1 . A value of zero is for no lateral load transfer. A maximum value of \pm 1 signifying total load on wheels on one side of the vehicle hence represents wheel(s) liftoff.

In a four-wheeled vehicle, a single wheel maintaining contact on either side would keep significant roll motion damping in effect. In a three-wheeled vehicle, only the axle having two wheels provides the roll motion damping; hence a single wheel liftoff is critical enough. In a delta configuration, braking unloads the rear wheels as shown in (5) and (6) reducing the threshold for lateral load transfer. Hence, for three-wheeled vehicles the following lateral load transfer ratio is suggested and is used in this research, depending only on wheel loads at the rear axle:

$$
\operatorname{LLTR}=\frac{F z_{r}-F z_{l}}{\left|F z_{r r}+F z_{r l}\right|},
$$

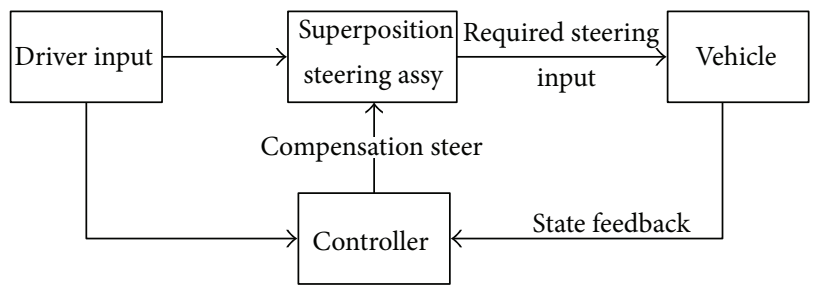

FIgURE 3: Layout suggested for superposition steering system for controller realization.

where $F z_{r r}$ and $F z_{r l}$ denote rear right and rear left vertical tire forces.

\section{Rollover Mitigation Controller}

A rollover mitigation controller based on steering alone is proposed. Sliding mode control is one of the many promising control techniques being used for vehicle control. Rollover mitigation controller in [17] uses reference lateral position and its derivative as the tracking error which are found by integrating lateral acceleration corresponding to threshold LLTR. Chen et al. [18] have proposed a sliding manifold using yaw rate following error, sideslip angle, and lateral acceleration. The controller exploits the yaw-roll coupling and differential braking is used as control input.

In this study roll angle $\phi_{\text {th }}$ corresponding to the target LLTR at steady state is set as limiting value. A superposition steering system as suggested by Joachim and Boerner [38] can be utilized for realization of this system and the layout is proposed as shown in Figure 3. This reduces the input required for the controller and only compensation steering input is required from the controller.

A direct mechanical linkage between steering wheel and road wheel along with feedback to the driver to an extent is maintained, conforming to existing road vehicle standards for steering systems. To limit the LLTR in finite time the sliding variable $\sigma$ is defined as follows:

$$
\sigma=\dot{e}+\alpha e,
$$

where $\alpha>0$ and $e=\phi_{\text {th }}-\phi$. The state feedback sliding mode control design is based upon roll dynamics equation (7), which in standard form including disturbances is written as follows:

$$
\ddot{\phi}=f(\phi, \dot{\phi})+g(z, \phi, \dot{\phi}) u+d(z, \phi, \dot{\phi}),
$$

where

$$
\begin{gathered}
f(\phi, \dot{\phi})=\frac{1}{I_{x x}}\left\{(h+z)\left[-\frac{2 \dot{x}\left(\dot{y}-l_{r} \dot{\psi}\right) C_{\alpha r}}{\left(\dot{x}^{2}-b^{2} \dot{\psi}^{2}\right)}\right]\right. \\
-2 b^{2}\left(K_{r} \phi+c_{r} \dot{\phi}\right)-(h+z) \\
\left.\cdot\left[\frac{\left(\dot{y}+l_{f} \dot{\psi}\right) C_{\alpha f}}{\dot{x}}\right]-\dot{\theta} \dot{\phi}\left(I_{z z}-I_{y y}\right)\right\}, \\
g=\frac{(h+z)}{I_{x x}} .
\end{gathered}
$$


This disturbance term $d(z, \phi, \dot{\phi})$ represents modeling uncertainties. The control law has two components as follows:

$$
u=u_{\mathrm{eq}}+u_{s}
$$

where $u_{\text {eq }}$ is the equivalent control and $u_{s}$ is the conventional switching control. The equivalent control is based upon the nominal model and is given by

$$
u_{\mathrm{eq}}=\frac{(-f(\phi, \dot{\phi})-\alpha \dot{\phi})}{g(z, \phi, \dot{\phi})} .
$$

The switching component $u_{s}$ is given by

$$
u_{s}=\rho \operatorname{sign}(\sigma) \text {, }
$$

where

$$
\rho=M+\kappa
$$

and $M$ is the bound on the disturbance term $d(z, \phi, \dot{\phi})$ that satisfies

$$
|d(z, \phi, \dot{\phi})| \leq M,
$$

for all $z, \phi, \dot{\phi}$ in the domain of interest.

The finite time convergence to the sliding surface is guaranteed by the positive constant $\kappa$ and the rate of convergence depends on its magnitude as discussed by Shtessel et al. [39].

An important consideration in the controller implementation is that the controller attempts to regulate the roll angle at the limiting value as soon it is activated. A threshold LLTR activates the controller. As roll angle value lags behind the LLTR by approximately 0.1 seconds with increasing LLTR reaching a level of critical value, the value of roll angle is still less than the critical value corresponding to steady state LLTR. If the controller is activated based on LLTR, it will try to immediately push the roll angle to set value increasing the LLTR overshoot significantly. The reference threshold in this condition is set as the current roll angle which is less than the threshold steady state roll angle corresponding to desired LLTR. The evolution of roll angle is thus restricted significantly. Furthermore the driver steering input is compared with the value of required steering input for maintaining the vehicle roll angle at threshold value. To provide for the switching control to work with less oscillations due to one sided fencing of the roll angle, the controller output value is used with correction even if it is up to 5\% less than steering input of the driver. This requires activation of controller prior to reaching the critical LLTR value. The switching relay part of the controller can have a larger gain for robustness requiring a larger operating range of controller output with respect to the driver input. It would result in delayed switch-off of the controller input even if the driver has turned the steering wheel back. To cater for this, the dynamic regularization of the relay part of the controller, sigmoid approximation of $\operatorname{sign}(\cdot)$ is used as shown in

$$
\operatorname{sign}(s)=\tanh \left(\frac{s}{\varepsilon}\right) \text {. }
$$

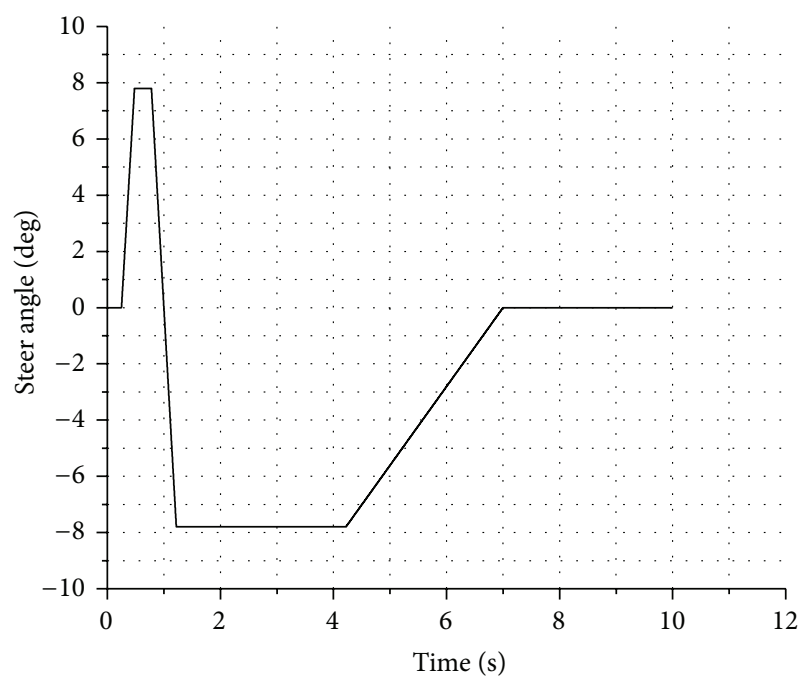

FIGURE 4: NHTSA Fishhook steering profile used for evaluating rollover propensity.

\section{Simulations}

In order to evaluate the efficacy of the proposed controller a high fidelity model of a three-wheeled vehicle is set up in CarSim software. This model includes nonlinear effects of roll center movement and tire force nonlinearities including tire relaxation length causing a further lag in response of the vehicle as discussed in Section 2 of this paper. The suspensions are also modeled using nonlinear dampers and stiffness which are typical of light vehicle suspension systems. The controller is coded as a level-1 S-function in Matlab/Simulink. NHTSA J-Turn test is used for evaluating the efficacy of the controller. A cosimulation is set up in Matlab/Simulink using simulation model of CarSim.

While assessing the available data on three-wheeled vehicles, it was observed that most of the untripped rollover accidents were caused by sudden large steering to avoid an obstacle on the road. The second major cause was overspeeding while cornering $[9,10]$. Considering the steering input severity pattern and available standards, NHTSA Fishhook maneuver was selected for evaluation of the proposed controller. A slowly increasing steering input at constant speed was given. A steering input corresponding to a lateral acceleration of $0.3 \mathrm{~g}$ is measured. This steering angle was scaled by a factor of 8 to account for maneuver severity and used as the amplitude of ramp steering on each direction. The angle was changed with a rate of 720 degrees per second during the first two ramps and a dwell period of three seconds after the steering reversal is maintained. The corresponding road wheel steering angle profile for a typical Asian threewheeled vehicle is as shown in Figure 4.

A maneuver entrance speed of $35 \mathrm{~km} / \mathrm{hr}$ is used. A threshold roll angle is set at a steady state LLTR of 0.8 arbitrarily considering a margin of safety. The LLTR with and without controller is shown in Figure 5. A value of 1 and -1 for LTTR indicates wheel liftoff, while the controller is able to maintain these values near 0.8 . The transients are managed 


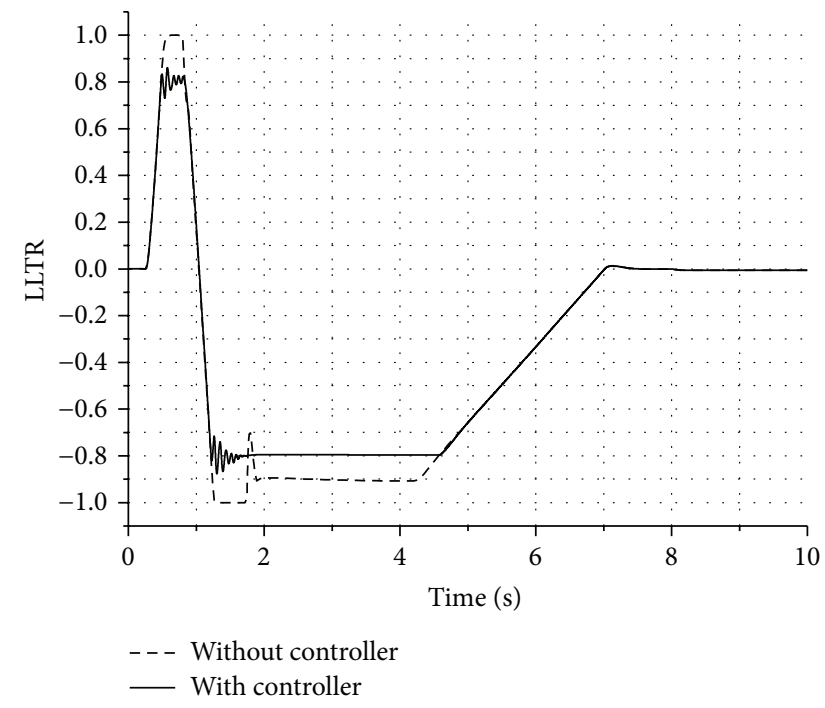

FIGURE 5: Evolution of LLTR during NHTSA Fishhook maneuver with and without controller. The maneuver entrance speed is set to correspond to wheel lift off during this maneuver without controller.

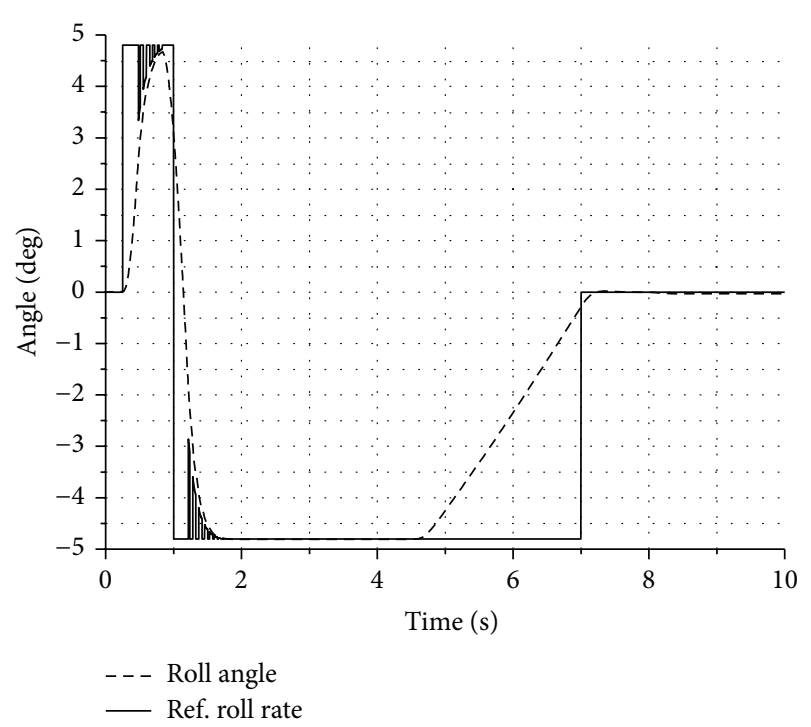

FIGURE 6: Proposed adapting reference roll angle and the actual roll angle during NHTSA Fishhook maneuver. The adaption is based on the value of LLTR.

in finite time as apparent in the dwell period from 1.7 to 4.2 seconds.

The overshoot of roll angle when reduced results in controller action which is restricted by the switching threshold on the lower side with a value of 0.75 . The adapted roll angle threshold is shown in Figure 6.

The adaption algorithm was used to compensate for the delay between the steering angle and the evolving roll angle. The value of roll angle reference for the controller is as follows:

$$
\phi_{\text {ref }}=\phi_{\text {set_point }}+\left(\phi_{\text {setpoint }}-\phi_{\text {actual }}\right) .
$$

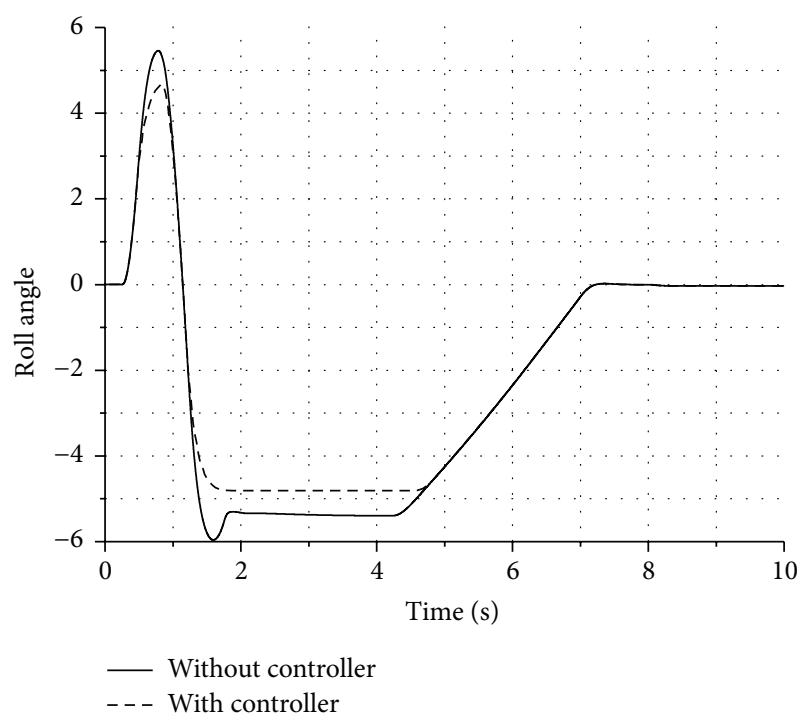

Figure 7: The evolution of roll angle of vehicle during NHTSA Fishhook maneuver with and without control.

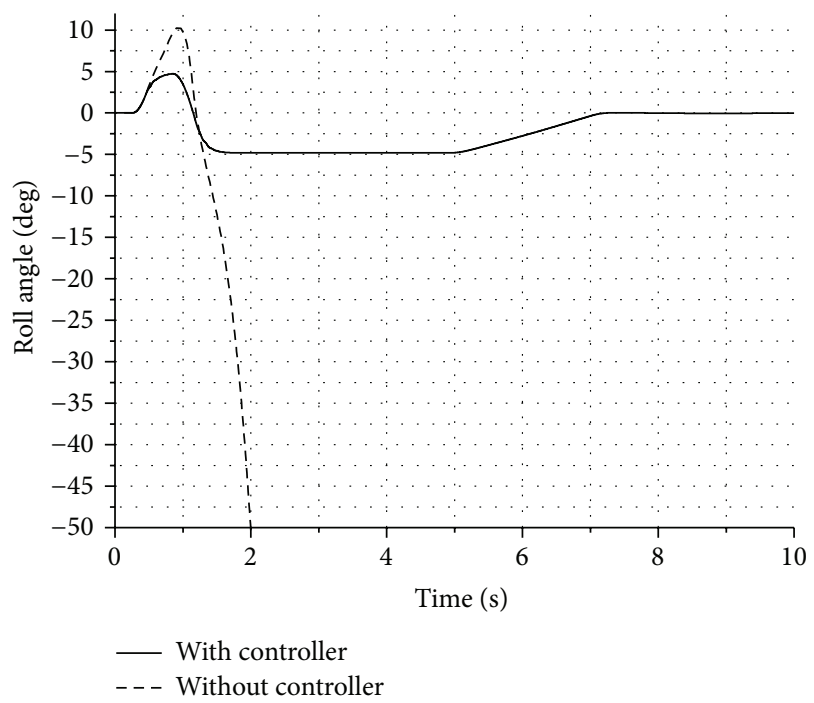

FIGURE 8: Roll angle evolution during Fishhook maneuver with an elevated speed of $38 \mathrm{~km} / \mathrm{hr}$. The controller was successful in limiting roll angle and mitigating rollover.

The value is updated only if the LLTR is greater than the threshold value which is 0.8 in this study and the actual roll angle is less than the set point steady state roll angle corresponding to the threshold LLTR. The evolution of roll angle with and without controller is shown in Figure 7. A threshold value for roll angle has been set at 4.8 degrees.

As the roll angle is the focus of the controller, it is effectively regulated at the set point value despite approximate model and model parameters. Road uncertainties, slight increase in vehicle speed, and lack of driving skills may result in rollover as indicated in Figure 8 indicating a roll angle profile with the same steering profile for a maneuver entrance speed of $38 \mathrm{~km} / \mathrm{hr}$. 


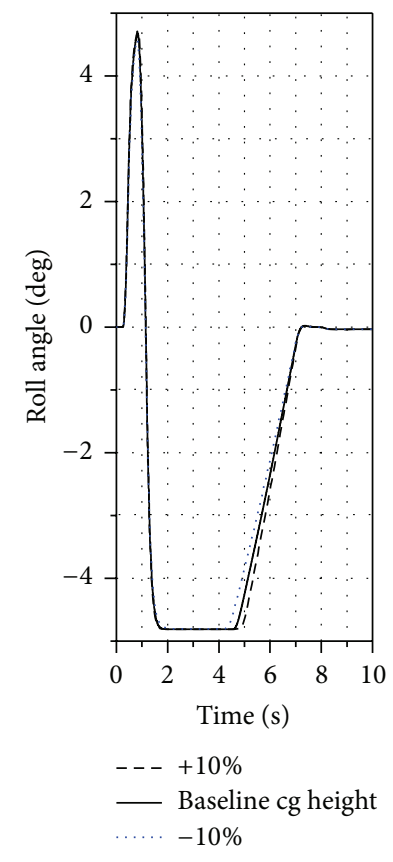

(a)

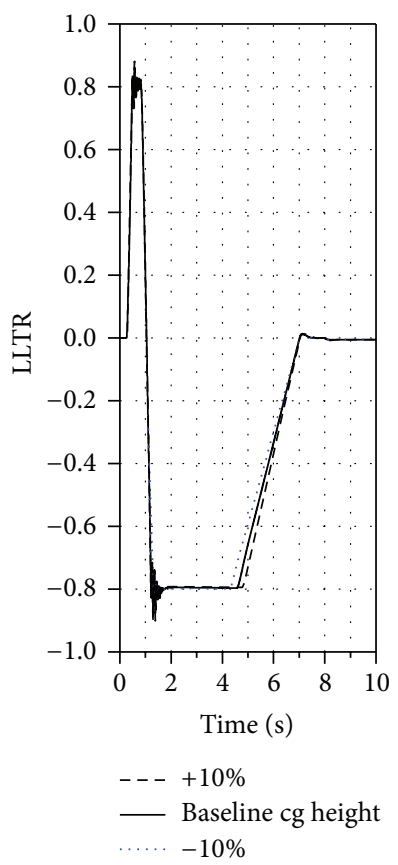

(b)

FIGURE 9: Evolution of roll angle and LLTR with 10\% variation in cg height. Controller was able to limit both with reasonable efficacy.

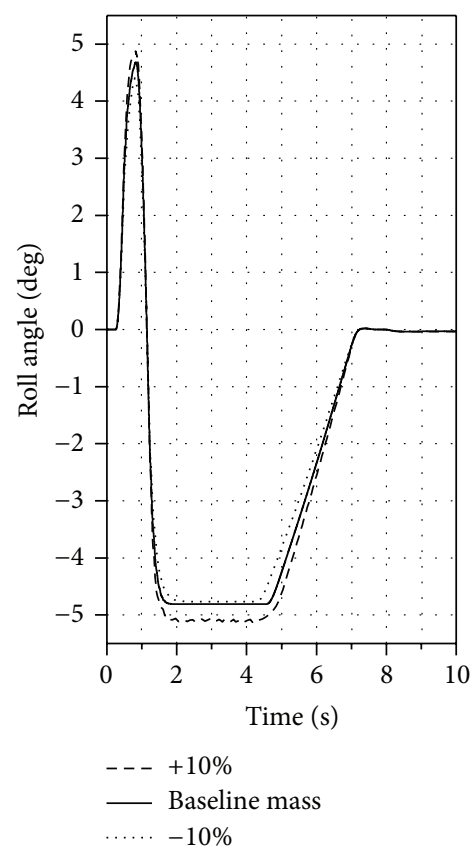

(a)

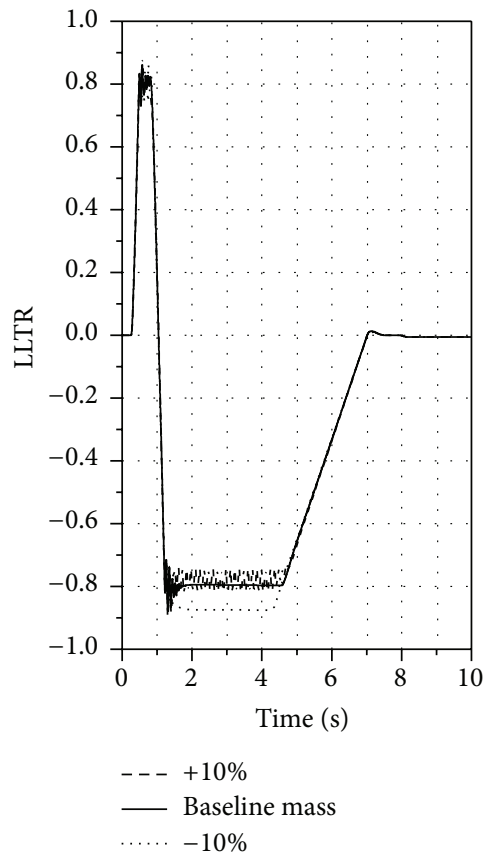

(b)

FIGURE 10: Evolution of roll angle and LLTR with $10 \%$ variation in vehicle mass. Controller was able to limit both with reasonable efficacy. With less mass, increase in roll angle results in more lateral load transfer.

As evident from Figure 8, the vehicle did not recover without controller after steering reversal with this slight increase in vehicle speed. However the controller was still able to limit the roll angle and LLTR despite this change in longitudinal speed signifying robustness. Next the controller was evaluated with $\pm 10 \%$ variations in height of $\mathrm{cg}$ from the ground and mass. The results are presented in Figures 9 and 10 , respectively.

These results show that the controller adapted better to cg height changes as compared to mass increase but still was able to maintain LLTR below one, that is, maintaining contact with ground. With additional mass the static deflection of 
suspension increases, reducing the suspension travel and at higher roll angles the bump stops start interacting, resulting in sudden change in stiffness. This results in oscillations about the steady state roll angle. The mass increase results in increased lateral force on the sprung mass for the same lateral acceleration. This results in an increase in roll angle. When the controller tries to regulate the roll angle, lateral load transfer is reduced, hence a better rollover mitigation. With reduced mass, the roll angle corresponding to the same maneuver reduces. When the controller tries to regulate it to a higher level, relatively more lateral load transfer results. This is evident from Figure 10. To overcome this, it is suggested that the controller should be tuned for lowest operational vehicle total mass. This would ensure robustness with load variations.

\section{Conclusion}

In this study a controller design methodology was presented for rollover mitigation of three-wheeled vehicles using active front steering. A low order, full vehicle model for roll dynamics was used with steering as a control input. A conventional sliding mode control with equivalent control was used. To account for the delay in vehicle response to control inputs an adaptive reference for roll angle has been proposed. The controller was evaluated for efficacy using a high fidelity simulation model of CarSim. The controller was able to mitigate rollover even in the presence of varying forward speed, cg, and mass variations. Vehicle loading increases the mass properties and cg height of a vehicle. Based on the performance of controller with reduced vehicle mass, it is recommended that the baseline mass properties used for controller design should correspond to the minimum anticipated operational loading instead of the nominal fully laden vehicle state. This would lead to maximum efficacy and robustness of the proposed controller.

\section{Nomenclature}

$\begin{array}{ll}x, y, z: \text { Displacements in body attached frame, } \mathrm{m} \\ \phi: & \text { Vehicle roll angle, rad } \\ \theta: & \text { Vehicle pitch angle, rad } \\ \psi: & \text { Vehicle yaw angle, rad } \\ l_{f}: & \text { Distance from cg to front axle, } \mathrm{m} \\ l_{r}: & \text { Distance from cg to rear axle, } \mathrm{m} \\ I_{x x}: & \text { Moment of inertia about roll axis, kg-m }{ }^{2} \\ I_{y y}: & \text { Moment of inertia about pitch axis, } \mathrm{kg}-\mathrm{m}^{2} \\ I_{z z}: & \text { Moment of inertia about yaw axis, } \mathrm{kg}-\mathrm{m}^{2} \\ b: & \text { Track width, } \mathrm{m} \\ h_{0}: & \text { Static height of center of gravity, } \mathrm{m} \\ P(t): & \text { Power applied at rear wheels, } \mathrm{W} \\ B x: & \text { Breaking force, } \mathrm{N} \\ \delta: & \text { Steering angle, rad } \\ F z: & \text { Vertical force at tire contact, } \mathrm{N} \\ F y: & \text { Tire lateral force, } \mathrm{N} \\ \alpha: & \text { Tire slip angle, rad. }\end{array}$

\section{Subscripts}

$f, r r, r l$ : Wheel locations front, rear right, and rear left.

\section{Conflict of Interests}

The authors declare that there is no conflict of interests regarding the publication of this paper.

\section{References}

[1] NHTSA, Initiatives to Address the Mitigation of Vehicle Rollover, National Highway Traffic Safety Administration, 2003.

[2] NHTSA, "Trends in the Static Stability Factor of Passenger Cars, Light Trucks, and Vans," NHTSA Technical Report DOT HS 809 868, NHTSA, 2005.

[3] P. G. Van Valkenburgh, R. H. Klein, and J. Kanianthra, “Threewheel passenger vehicle stability and handling," SAE Technical Paper 820140, 1982.

[4] A. Raman, J. S. Rao, and S. R. Kale, "Overturning stability of three wheeled motorized vehicles," Vehicle System Dynamics, vol. 24, no. 2, pp. 123-144, 1995.

[5] T. R. Gawade, S. Mukherjee, and D. Mohan, "Rollover propensity of three-wheel scooter taxis," SAE Technical Paper Series 2004-01-1622, 2004.

[6] S. Mukherjee, D. Mohan, and T. R. Gawade, "Three-wheeled scooter taxi: a safety analysis," Sadhana, vol. 32 , no. 4 , pp. 459478, 2007.

[7] G. J. Forkenbrock, W. R. Garrott, M. Heitz, and B. C. O. Harra, Experimental Examination of Test Maneuvers That May Induce On-Road, Untripped Light Vehicle Rollover, 2003.

[8] Government of India, Road Accidents in India 2010, Government of India, New Delhi, India, 2010.

[9] Government of India, Road Accidents in India 2011, Government of India, New Delhi, India, 2011.

[10] Government of India, Road Accidents in India 2009, New Delhi, India, 2009.

[11] A. Mani, M. Pai, and R. Aggarwal, "Sustainable urban transport policy in India: focus on Autorickshaw sector," Journal of the Transportation Research Board, vol. 2317, pp. 104-110, 2012.

[12] J. C. Huston, B. J. Graves, and D. B. Johnson, "Three wheeled vehicle dynamics," Tech. Rep. SAE Technical Series Paper no. 820139, 1982.

[13] S. Yim, "Design of a preview controller for vehicle rollover prevention," IEEE Transactions on Vehicular Technology, vol. 60, no. 9, pp. 4217-4226, 2011.

[14] J. N. Dang, "Preliminary results analyzing the effectiveness of electronic stability control (ESC) systems," 2004.

[15] M. A. R. Licea and I. Cervantes, "Robust switched predictive braking control for rollover prevention in wheeled vehicles," Mathematical Problems in Engineering, vol. 2014, Article ID 356250, 12 pages, 2014.

[16] Y. Suetake, M. Oya, P. Shu, and J. Zhuo, "Adaptive rollover prevention controller for driver-vehicle systems," Artificial Life and Robotics, vol. 19, no. 1, pp. 9-15, 2014.

[17] H. Imine, L. M. Fridman, and T. Madani, "Steering control for rollover avoidance of heavy vehicles," IEEE Transactions on Vehicular Technology, vol. 61, no. 8, pp. 3499-3509, 2012.

[18] B.-C. Chen, C.-C. Yu, W.-F. Hsu, and M.-F. Lo, "Design of electronic stability control for rollover prevention using sliding mode control," International Journal of Vehicle Design, vol. 56, no. 1-4, pp. 224-245, 2011.

[19] D. J. M. Sampson and D. Cebon, "Active roll control of single unit heavy road vehicles," Vehicle System Dynamics, vol. 40, no. 4, pp. 229-270, 2003. 
[20] B. L. J. Gysen, J. J. H. Paulides, J. L. G. Janssen, and E. A. Lomonova, "Active electromagnetic suspension system for improved vehicle dynamics," IEEE Transactions on Vehicular Technology, vol. 59, no. 3, pp. 1156-1163, 2010.

[21] H. Yu, L. Güvenc, and Ü. Özgüner, "Heavy duty vehicle rollover detection and active roll control," Vehicle System Dynamics: International Journal of Vehicle Mechanics and Mobility, vol. 46, no. 6, pp. 451-470, 2008.

[22] J. Tjonnas and T. A. Johansen, "Stabilization of automotive vehicles using active steering and adaptive brake control allocation," IEEE Transactions on Control Systems Technology, vol. 18, no. 3, pp. 545-558, 2010.

[23] S. C. Peters, J. E. Bobrow, and K. Iagnemma, "Stabilizing a vehicle near rollover: an analogy to cart-pole stabilization," in Proceedings of the IEEE International Conference on Robotics and Automation (ICRA '10), pp. 5194-5200, May 2010.

[24] M. M. Islam and C. Ha, "Road vehicle rollover avoidance using active steering controller," in Proceedings of the 14th International Conference on Computer and Information Technology (ICCIT '11), pp. 298-302, Dhaka, Bangladesh, December 2011.

[25] J. Yoon, W. Cho, B. Koo, and K. Yi, "Unified chassis control for rollover prevention and lateral stability," IEEE Transactions on Vehicular Technology, vol. 58, no. 2, pp. 596-609, 2009.

[26] J. Yoon, W. Cho, J. Kang, B. Koo, and K. Yi, "Design and evaluation of a unified chassis control system for rollover prevention and vehicle stability improvement on a virtual test track," Control Engineering Practice, vol. 18, no. 6, pp. 585-597, 2010.

[27] B. Zhang and L. Tong, "Modeling and simulation of integrated steering and braking control for vehicle active safety system," International Journal of Image, Graphics and Signal Processing, vol. 3, no. 2, p. 1, 2011.

[28] D. Piyabongkarn, T. Keviczky, and R. Rajamani, "Active direct tilt control for stability enhancement of a narrow commuter vehicle," International Journal of Automotive Technology, vol. 5, no. 2, pp. 77-78, 2004.

[29] M. Barker, B. Drew, J. Darling, K. A. Edge, and G. W. Owen, "Steady-state steering of a tilting three-wheeled vehicle," Vehicle System Dynamics, vol. 48, no. 7, pp. 815-830, 2010.

[30] S. Kidane, R. Rajamani, L. Alexander, P. J. Starr, and M. Donath, "Development and experimental evaluation of a tilt stability control system for narrow commuter vehicles," IEEE Transactions on Control Systems Technology, vol. 18, no. 6, pp. 1266-1279, 2010.

[31] T. Shim and C. Ghike, "Understanding the limitations of different vehicle models for roll dynamics studies," Vehicle System Dynamics, vol. 45, no. 3, pp. 191-216, 2007.

[32] N. Zhang, G.-M. Dong, and H.-P. Du, "Investigation into untripped rollover of light vehicles in the modified fishhook and the sine maneuvers. Part I: vehicle modelling, roll and yaw instability," Vehicle System Dynamics, vol. 46, no. 4, pp. 271-293, 2008.

[33] T. R. Gawade, S. Mukherjee, and D. Mohan, "Six-degree-offreedom three-wheeled-vehicle model validation," Proceedings of the Institution of Mechanical Engineers, Part D: Journal of Automobile Engineering, vol. 219, no. 4, pp. 487-498, 2005.

[34] T. R. Gawade, S. Mukherjee, and D. Mohan, "Wheel lift-off and ride comfort of three-wheeled vehicle over bump," Journal of the Institution of Engineers, Part MC: Mechanical Engineering Division, vol. 85, pp. 78-87, 2004.
[35] A. Hac, "Rollover stability index including effects of suspension design," in Proceedings of the SAE 2002 World Congress \& Exhibition, No. 724, Detroit, Mich, USA, 2002.

[36] J. Jung, T. Shim, and J. Gertsch, "A vehicle roll-stability indicator incorporating roll-center movements," IEEE Transactions on Vehicular Technology, vol. 58, no. 8, pp. 4078-4087, 2009.

[37] R. J. Whitehead, W. E. Travis, D. D. M. D. M. Bevly, and G. T. Flowers, "A study of the effect of various vehicle properties on rollover propensity," in Proceedings of the SAE Automotive Dynamics, Stability \& Controls Conference and Exhibition, pp. 3381-3386, 2004.

[38] F. Joachim and J. Boerner, "Superposition-drive for steering system," US Patent No. 20100331133A12010.

[39] Y. Shtessel, C. Edwards, L. Fridman, and A. Levant, Sliding Mode Control and Observation, Springer, New York, NY, USA, 2014. 


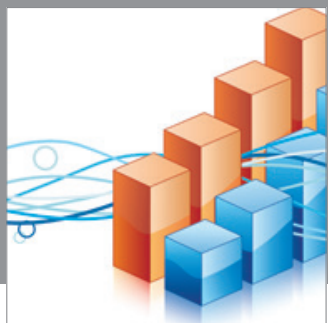

Advances in

Operations Research

mansans

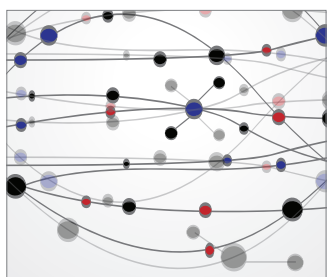

The Scientific World Journal
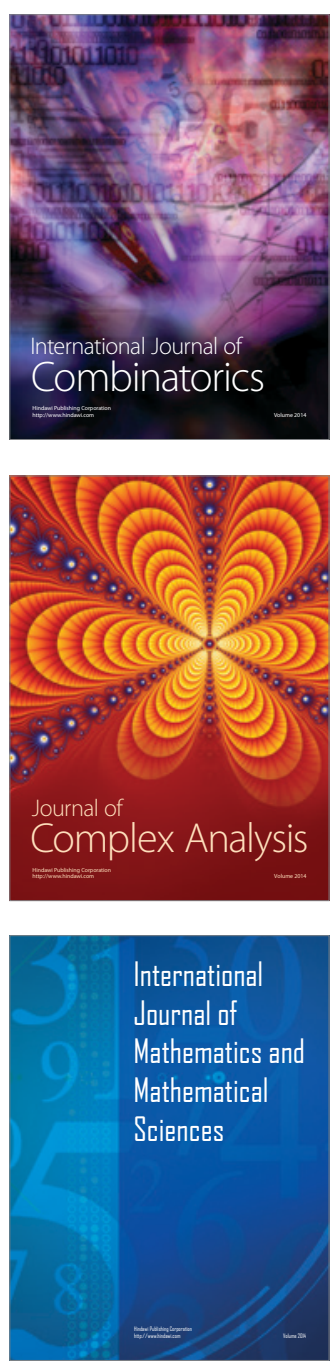
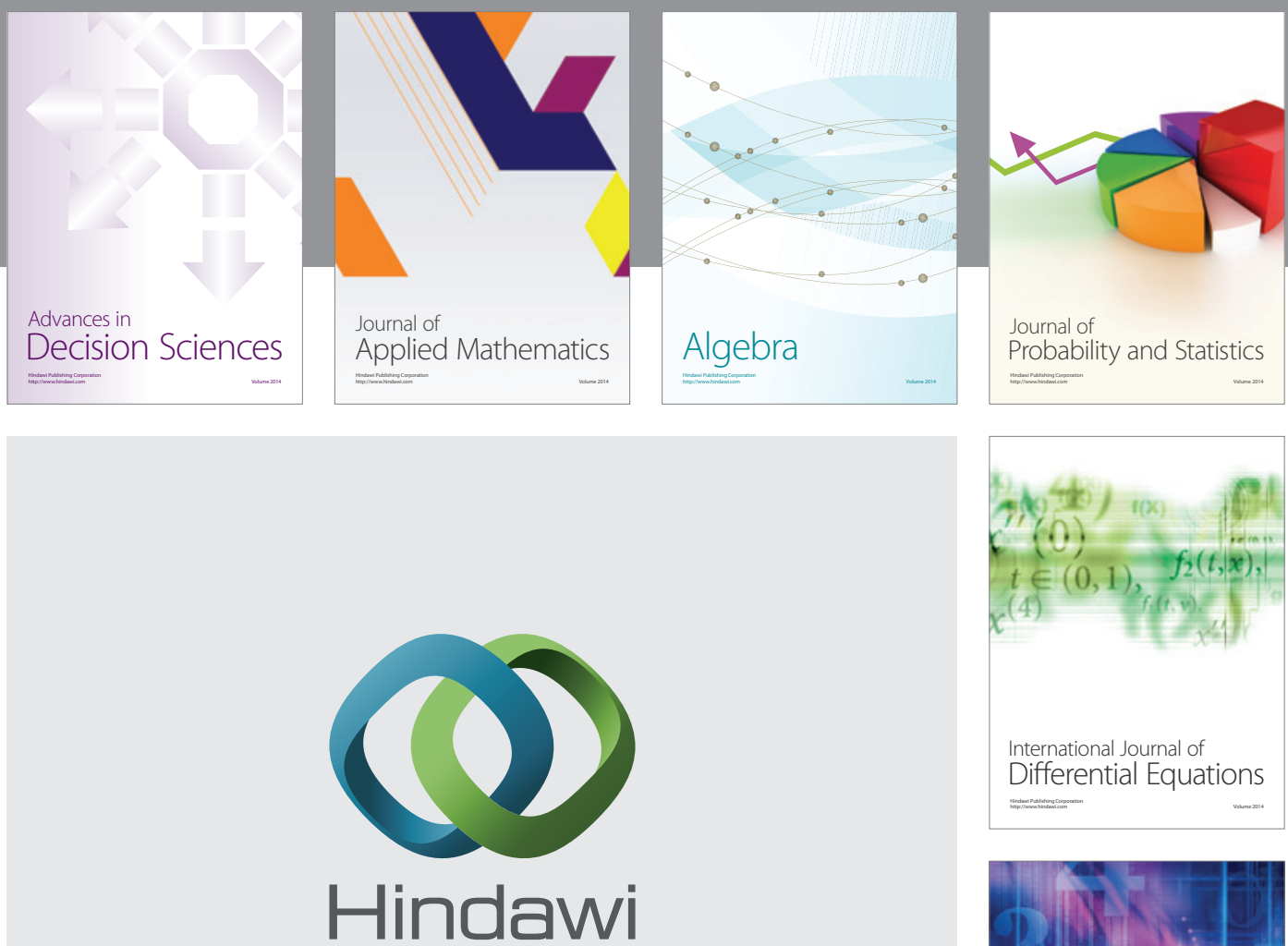

Submit your manuscripts at http://www.hindawi.com
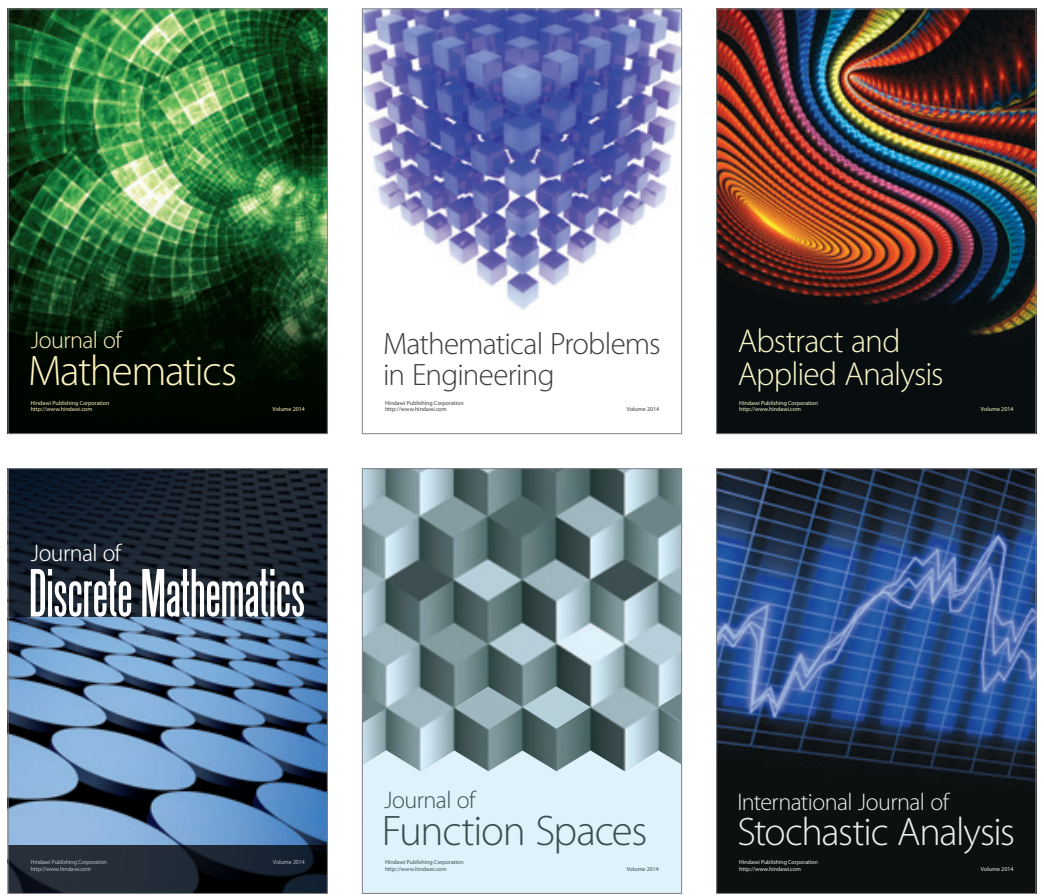

Journal of

Function Spaces

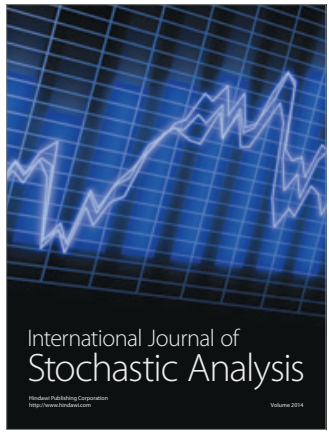

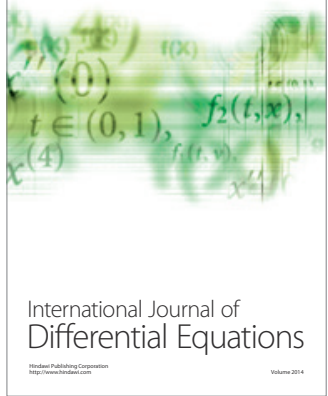
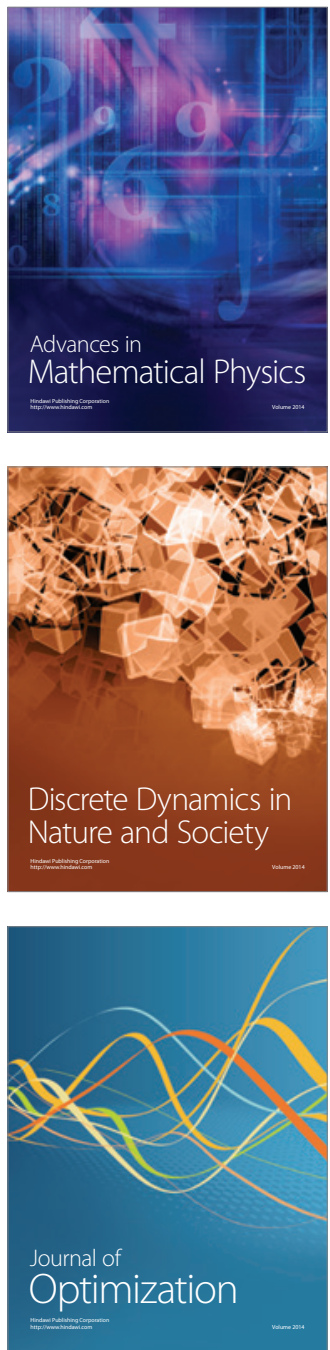